Original Research Paper

\title{
A Simple Model of Gauged Lepton and Baryon Charges
}

\section{P.V. Dong and H.N. Long}

Institute of Physics, Vietnam Academy of Science and Technology, 10 Dao Tan, Ba Dinh, Hanoi, Vietnam

Article history

Received: 05-12-2014

Revised: 03-01-2015

Accepted: 26-05-2015

Corresponding Author:

P.V. Dong

Institute of Physics, Vietnam

Academy of Science and

Technology, 10 Dao Tan, Ba

Dinh, Hanoi, Vietnam

Email: pvdong@iop.vast.ac.vn

\begin{abstract}
We propose a simple extension of the standard model with lepton and baryon numbers gauged by introducing only two leptoquarks $\left(\mathrm{j}^{+1 / 2}, \mathrm{k}^{-1 / 2}\right)$ to the fermion content. As natural consequences of the anomaly cancelation, the right-handed neutrinos present and the number of observed fermion families equals to the number of fundamental colors, which is three. To avoid stabilized colored-leptoquarks, two scalar doublets $\phi, \chi$ are introduced which couple the leptoquarks to ordinary quarks. The new gauge bosons $Z_{L}^{\prime}, Z_{B}^{\prime}$ gain masses via the vacuum values of scalars $S_{L}, S_{B}$ which are only charged under the $U(1)_{L}$, $\mathrm{U}(1)_{\mathrm{B}}$ symmetries, respectively. Furthermore, due to the spontaneous symmetry breaking of these charges, the neutrinos obtain small masses via type I seesaw mechanism while the baryogenesis can be generated in several cases. Constraints coming from flavor changing, stabilization of anomalously electriccharged scalars and collision phenomenology are also obtained.
\end{abstract}

Keywords: $12.60 . \mathrm{Cn}, 11.30 . \mathrm{Fs}, 14.65 . \mathrm{Jk}$

\section{Introduction}

It has been evidenced that the Lepton charge (L) and the Baryon charge (B) belong to exact symmetries up to very high scales much beyond $\mathrm{TeV}$ scale (Nakamura et al., 2010). They may be only violated at the scales as of the grand unification theories such as that of the type I seesaw mechanism to explain the smallness of neutrino masses and that to describe the stability of proton in the proton decay question. It is therefore believed that these charges are of exact symmetries but spontaneously broken as the gauge symmetries. The possible phenomenologies of such theories with spontaneous symmetry breaking at the $\mathrm{TeV}$ scale have been recently attracted much attention (Foot et al., 1989; Carone and Murayama, 1995; Perez and Wise, 2010; Dulaney et al., 2011; Chao, 2010; Ko and Omura, 2011).

If the lepton and baryon charges respectively behave as their own gauge symmetries, it is natural to extend the Standard Model (SM) gauge symmetry into a larger group $\mathrm{G}=\mathrm{SU}(3) \otimes \mathrm{SU}(2) \otimes \mathrm{U}(1)_{\mathrm{Y}} \otimes \mathrm{U}(1)_{\mathrm{L}} \otimes \mathrm{U}(1)_{\mathrm{B}}$, where the last two factors are the gauge groups of the lepton and baryon charges, respectively. The consistent condition of the model requires that all the anomalies associated with $G$ must be canceled. However, in the literature the models of lepton and baryon charges gauged are actually complicated in the fermionic contents because they include many exotic particles as a result to cancel the anomalies. Also, the seesaw mechanism responsible for generating the neutrino small masses is quite complex due to the contributions of a large spectrum of neutral leptons (Foot et al., 1989; Carone and Murayama, 1995; Perez and Wise, 2010; Dulaney et al., 2011; Chao, 2010 and Ko and Omura, 2011). Taking an example, a fourth family of leptons and a fourth family of quarks which are replications of the ordinary fermion families including the right-handed neutrinos were introduced into the SM, in which the fourth family exotic leptons have $\mathrm{L}=(+/-) 3$ and the exotic quarks having $\mathrm{B}=(+/-) 1$. The sign either plus or minus will appropriately take place depending on which chirality left or right assigned on the fermion multiplets, respectively (Perez and Wise, 2010; Dulaney et al., 2011). Let us remind the reader that in these models the right-handed neutrinos of all the lepton families are in general not required on the background of the anomaly cancelation. In the following we propose simpler fermionic contents which are free from all the anomalies.

The idea is that the leptonic and baryonic anomalies as stored in the SM particle content can be removed by introducing a single family of only a fermion kind of the so-called leptoquarks with appropriate quantum numbers. Hence, the leptoquarks are colored like quarks, but they possess both lepton and baryon charges with ordinary values like those of the well-known particles-leptons and hadrons. The leptonic content in our model is thus minimal since it only includes the ordinary right-handed neutrinos, as played in the usual type I seesaw mechanism, emerging as a natural requirement of the gravity-anomaly cancelation. From vanishing of another anomaly, the number of experimentally-observed fermion families as in 
the SM is found to be related to the number of the fundamental colors, which equals to three. The leptoquarks have abnormal electric charges and do not mix with the ordinary quarks. Therefore, there is no tree-level Flavor Changing Neutral Current (FCNC), which is a new observation of this work. To avoid unwanted stabilized colored-particles, two new scalar doublets are included which couple the leptoquarks to the ordinary quarks. Consequently, the model contains a stable charged scalar (with an anomalous electriccharge) due to the charge conservation. The new gauge symmetries $\mathrm{U}(1)_{\mathrm{L}}$ and $\mathrm{U}(1)_{\mathrm{B}}$ are spontaneously broken due to two new singlet scalars which are only charged under these symmetries.

Advantages of the model such as simplicity in the seesaw mechanism responsible for the neutrino masses, the generation of baryon number asymmetry coming from several sources, the phenomenological processes due to the contributions of the leptoquarks, new gauge bosons, new scalars as well as the bounds on the model's parameters via flavor changing are shown. The collider and cosmological constraints on the stable anomalously-charged scalar are considered. We also indicate that the proton is stabilized due to the new gauge symmetries in spite of the fact that the lepton and baryon charges are broken at the $\mathrm{TeV}$ scale. Thus, this setup disregards the postulate of a large desert between the weak scale and the $\mathrm{U}(1)_{\mathrm{L}, \mathrm{B}}$ scales. The validate of the model as well as the experimental signatures of some new particles will be discussed.

The rest of this article is organized as follows. In the next section, Section. II, we construct the model by stressing on new fermionic contents. Section III is devoted to scalar particles, Yukawa interactions, fermion masses and some remarkable phenomenologies. In Sec. IV, we present constraints on the new physics such as FCNCs in the quark and lepton sectors, stable scalar, baryogenesis and effects of the new particles including their possible processes and productions at the existing and future colliders. We summarize our results and make conclusions in Section V.

\section{The Model}

To cancel the anomalies associated with the gauge symmetry $\mathrm{G}=\mathrm{SU}(3) \otimes \mathrm{SU}(2) \otimes \mathrm{U}(1)_{\mathrm{Y}} \otimes \mathrm{U}(1)_{\mathrm{L}} \otimes \mathrm{U}(1)_{\mathrm{B}}$, we introduce into the SM particle content a single kind of only colored-fermions $\mathrm{j}$ and $\mathrm{k}$ with $\mathrm{L}=-1, \mathrm{~B}=-1$ and electric charges $q_{j}=1 / 2, q_{k}=-1 / 2$, called leptoquarks. In addition, the right-handed neutrinos corresponding to the SM lepton families will be included in order to cancel the gravity anomaly. The particle content in our model under $\mathrm{G}$ transform as:

$\begin{array}{ll}\text { SM leptons } & \psi_{a L} \equiv\left(v_{a L} e_{a L}\right) \sim(1,2,-1 / 2,1, \\ & 0), e_{a R} \sim(1,1,-1,1,0) \\ \text { SM quarks } & Q_{a L} \equiv\left(u_{a L} d_{a L}\right) \sim(3,2,1 / 6,0,1 / 3),\end{array}$

$u_{a R} \sim(3,1,2 / 3,0,1 / 3)$,
$d_{a R} \sim(3,1,-1 / 3,0,1 / 3)$

Right-handed neutrinos $v_{a R} \sim(1,1,0,1,0)$

Leptoquarks

$$
\begin{aligned}
F_{L} & \equiv\left(j_{L} k_{L}\right) \sim(3,2,0,-1,-1), \\
j_{R} & \sim(3,1,1 / 2,-1,-1), \\
k_{R} & \sim(3,1,-1 / 2,-1,-1)
\end{aligned}
$$

where, $\mathrm{a}=1,2,3$ is family index and the values in the parentheses denote quantum numbers based on the $(S U(3)$, $\left.S U(2), U(1)_{Y}, U(1)_{L}, U(1)_{B}\right)$ symmetries, respectively.

One can check that all the anomalies are canceled, for examples:

$$
\begin{aligned}
& {[S U(3)]^{2} U(1)_{Y}=0,[S U(2)]^{2} U(1)_{Y}=0,} \\
& {[\text { Gravity }]^{2} U(1)_{Y}=0,}
\end{aligned}
$$

$\left[U(1)_{Y}\right]^{3}=0,[S U(3)]^{2} U(1)_{L}=0,[S U(3)]^{2} U(1)_{B}=0$,

$[S U(2)]^{2} U(1)_{L}=(3 \sim$ number of SM lepton families $) \times(1)$

$+(3 \sim$ number of fundamental colors $) \times(-1)=0$

$[S U(2)]^{2} U(1)_{B}=(3 \sim$ number of SM quark families $)$

$\times(3 \sim$ number of fundamental colors $) \times(1 / 3)$

$+(3 \sim$ number of fundamental colors $) \times(-1)=0$,

$\left[U(1)_{Y}\right]^{2} U(1)_{L}=3 \times(-1 / 2)^{2} \times(1+1)$
$-3 \times(-1)^{2} \times 1+3 \times(0)^{2} \times(-1-1)$
$-3 \times(1 / 2)^{2} \times(-1)-3 \times(-1 / 2)^{2} \times(-1)=0$
$\left[U(1)_{Y}\right]^{2} U(1)_{B}=3 \times 3 \times(1 / 6)^{2}$
$\times(1 / 3+1 / 3)-3 \times 3 \times(2 / 3)^{2} \times(1 / 3)$
$-3 \times 3 \times(-1 / 3)^{2} \times(1 / 3)+3 \times(0)^{2} \times(-1-1)$
$-3 \times(1 / 2)^{2} \times(-1)-3 \times(-1 / 2)^{2} \times(-1)=0$,
$[\text { Gravity }]^{2} U(1)_{L}$
$=3 \times(1 \sim$ lepton-charge of left-handed neutrino $)$
$-3 \times(1 \sim$ lepton-charge of right-handed neutrino $)=0$.

The last equation shows that the right-handed neutrinos are required. If one supposes that the leptoquarks have no exotic lepton charges as we took $L=-1$, then the number of ordinary lepton families is equal to the number of the fundamental colors (as we can see above from the $[S U(2)]^{2} U(1)_{L}$ anomaly cancelation). Otherwise, the family number will be a multiple of the color number. The similar one with the baryonic anomaly implies that the number of the observed quark families is also related to the color number. The leptoquarks possessing $B=-1$ as we put are in order to cancel the baryonic anomalies. Note that in the framework of the 3-3-1 models, the family 
number achieved has a different origin, which is a consequence of the new weak-isospin symmetry $S U(3)_{L}$ with a corresponding enlargement of the lepton and quark representations (see, for recent works, Kelso et al., 2014; Cogollo et al., 2014; Dong et al., 2014; 2013; 2012). It is noteworthy that the $[S U(2)]^{2} U(1)_{Y}$ anomaly was removed because of $Y\left(F_{L}\right)=0$ and this is why we assumed $q_{j}=-q_{k}=1 / 2$. In summary, the baryonic and leptonic anomalies as stored in the SM particle content are all canceled due to the presence of just two leptoquarks $(j, k)$.

It is also noted that we can have another fermion content if one reverses the chirality of leptoquarks, simultaneously changes their sign of baryon and lepton charges $(L=+1, B=+1): F_{R}=\left(j_{R} k_{R}\right) \sim(3,2,0,1,1), j_{L} \sim$ $(3,1,1 / 2,1,1)$ and $k_{L} \sim(3,1,-1 / 2,1,1)$. In the following, we consider the case with the first fermionic content as given in the table above. The scalar sector will be introduced as usual to generate the mass for the particles and to make the model viable.

\section{Scalars, Yukawa Interactions and Related Phenomena}

The Higgs doublet of the SM transforms as follows $H=\left(H^{+} H^{0}\right) \sim(1,2,1 / 2,0,0)$. We see that $H$ does not couple the leptoquarks and the ordinary quarks together because of the $U(1)_{Y, L, B}$ gauge invariance. The Yukawa interactions are:

$$
\begin{gathered}
-L_{H}=h_{a b}^{e} \bar{\psi}_{a L} H e_{b R}+h_{a b}^{v} \bar{\psi}_{a L} i \sigma_{2} H^{*} v_{b R} \\
+h_{a b}^{d} \bar{Q}_{a L} H d_{b R}+h_{a b}^{u} \bar{Q}_{a L} i \sigma_{2} H^{*} u_{b R} \\
+h^{k} \bar{F}_{L} H k_{R}+h^{j} \bar{F}_{L} i \sigma_{2} H^{*} j_{R} \\
+ \text { h.c. }
\end{gathered}
$$

One can check that all the ordinary fermions, charged leptons and quarks, gain consistent masses similarly to the case of the SM. The neutrinos $v_{L}$ and $v_{R}$ at this step obtain Dirac masses as:

$$
M_{D}=h^{v}\left\langle H^{0}\right\rangle
$$

where, $\left\langle H^{0}\right\rangle=174 \mathrm{GeV}$ is the Vacuum Expectation Value $(\mathrm{VEV})$ of $H^{0}$ and $\mathrm{M}_{\mathrm{D}}$ is a $3 \times 3$ matrix in a and $\mathrm{b}$ family indices. It is easily checked that the leptoquarks do not mix with the ordinary quarks and have masses proportional to $\left\langle H^{0}\right\rangle$. They should be uncharacteristically heavy like top quark, but differently from the top quark because these particles possess the unusual charges such as lepton, baryon and electricity.

Next, let us introduce a scalar singlet $\mathrm{S}_{\mathrm{L}} \sim(1,1,0,-2,0)$ which couples to the right-handed neutrinos $v_{R}$. The Yukawa interaction is then:

$$
-L_{S_{L}}=\frac{1}{2} \lambda_{a b}^{v} \bar{v}_{a R}^{c} v_{b R} S_{L}+\text { h.c. }
$$

When $S_{L}$ develops a VEV, it provides not only Majorana mass for $v_{\mathrm{R}}$ :

$$
M_{R}=\lambda^{v}\left\langle S_{L}\right\rangle
$$

which is also a $3 \times 3$ matrix in family indices, but also a necessary mass for the $U(1)_{L}$ gauge boson $Z_{L}^{\prime}: M_{Z_{L}^{\prime}}=g_{L}\left\langle S_{L}\right\rangle$ where $g_{L}$ is the $U(1)_{L}$ gauge coupling constant. Let us note that the lepton charge is spontaneously broken by the $S_{L}$ scalar. To be consistent with the effective theory, we should impose $\left\langle S_{L}\right\rangle \gg\left\langle H^{0}\right\rangle$.

An interesting result from our proposal is that three active neutrinos $\left(\sim v_{L}\right)$ gain masses via a type I seesaw mechanism similar to the simplest seesaw extension of the SM:

$M_{v}^{e f f}=-M_{D} M_{R}^{-1} M_{D}^{T}$

which is quite different from the previous proposals (Perez and Wise, 2010; Chao, 2010). This is also a simple one like the fermionic content in our model.

To avoid having stable colored particles we will introduce the following scalar doublets charged under $U(1)_{L, B}$ that couples the leptoquarks to the ordinary quarks:

$$
\begin{aligned}
& \phi=\left(\phi^{+7 / 6} \phi^{+1 / 6}\right) \sim(1,2,2 / 3,1,4 / 3), \\
& \chi=\left(\chi^{+1 / 6} \chi^{-5 / 6}\right) \sim(1,2,-1 / 3,1,4 / 3)
\end{aligned}
$$

The Yukawa interactions are then:

$$
\begin{aligned}
& -L_{\phi \chi}=h_{a}^{\prime k} \bar{Q}_{a L} \phi k_{R}+h^{\prime u} \bar{F}_{L} i \sigma_{2} \phi^{*} u_{a R} \\
& +h_{a}^{\prime j} \bar{Q}_{a L} \chi j_{R}+h_{a}^{\prime d} \bar{F}_{L} i \sigma_{2} \chi^{*} d_{a R}+\text { h.c. }
\end{aligned}
$$

The leptoquarks can now decay into a scalar and SM ordinary quark. It is noteworthy that in our model the $\phi$ and $\chi$ scalars could not develop a VEV because of electric charge conservation, which is also a new feature and in contradiction to (Perez and Wise, 2010). Consequently, the leptoquarks do not mix in mass with the ordinary quarks (notice also that they are different in electric charges) and the FCNCs at the tree level never appear.

Let us note that the scalars $\chi$ and $\phi$ have the anomalous fractional electric charges, unlike the ordinary quarks. With the electric charge conservation, this implies that at least one of the scalars is absolutely stable. Unfortunately, it maynot be a dark matter since it has an electric charge. See also (Rujula et al., 1990; Dimopoulos et al., 1990; and so forth, up to LEP SUSY Working Group) for similar matters related to long-lived massive charged particles, but the stability of these particles origins differently from ours. 
Finally, the baryon charge should be spontaneously broken too. This may be achieved by another scalar singlet $S_{B}$ charged under $U(1)_{B}$. Then, the $U(1)_{B}$ gauge boson $Z_{B}^{\prime}$ will gain a mass proportional to $\left\langle S_{B}\right\rangle$. The consistent condition with the effective theory also implies $\left\langle S_{B}\right\rangle \gg\left\langle H^{0}\right\rangle$.

The scalar potential that consists of doublets $H, \phi$, $\chi$ and singlets $S_{L}, S_{B}$ can be easily written. Here we notice that all the charged scalars as contained in the doublets do not mix among them and with the others and by themselves become mass eigenstates.

\section{Constraints on the New Physics}

\section{Flavor Changing}

First we consider the flavor violation in the quark sector. As mentioned above, there is no FCNC at the tree level because of the gauge symmetry. We also know that the ordinary quarks have couplings to the leptoquarks. This will lead to the FCNC processes in the ordinary quark sector at one loop level via exchange of the leptoquarks. In this work, we consider the decay $b \rightarrow s \gamma$ and processes associated with $K^{0}-\bar{K}^{0}$ mixing (the mixings $D^{0}-\bar{D}^{0}, B^{0}-\bar{B}^{0}$ and decays $s \rightarrow d \gamma$, $b \rightarrow d \gamma, t \rightarrow d \gamma$ and so on can be similarly calculated).

The contributions to the $K^{0}-\bar{K}^{0}$ mixing come from the box diagrams as shown in Fig. 1. After integrating out the heavy particles with a characteristic mass scale $M$, the amplitude is proportional to $\left[\left(h^{\prime}\right)^{4} /\left(16 \pi^{2} M^{2}\right)\right]\left(\bar{d}_{L, R} \gamma_{\mu} s_{L, R}\right)\left(\bar{d}_{L, R} \gamma^{\mu} s_{L, R}\right)+$ h.c., where $\left(h^{\prime}\right)^{4}$ is some product of $h^{\prime d}, h^{\prime k}$, or $h^{\prime j}$ and appropriate quark mixing matrix elements. For $M$ of order $100 \mathrm{GeV}$, it is negligible provided $h^{\prime}<10^{-2}$, which is similar to (Perez and Wise, 2010).

The contributions to $b \rightarrow s \gamma$ are given in Fig. 2. In order to arrive at the branching ratio $\operatorname{Br}(b \rightarrow s \gamma)$ we divide as usual the decay width $\Gamma(b \rightarrow s \gamma)$ by the theoretical expression for the semileptonic decay width $\Gamma(b \rightarrow X e v)$ and multiply this ratio with the measured semileptonic branching ratio $\mathrm{Br}(b \rightarrow \mathrm{Xev}) \simeq 0.1$ (Nakamura et al., 2010):

$B r(b \rightarrow s \gamma)=\frac{\Gamma(b \rightarrow s \gamma)}{\Gamma(b \rightarrow X e v)} B r(b \rightarrow X e v)$

The semileptonic decay width (see, for example, Greub et al., 1996) is:

$$
\begin{aligned}
\Gamma(b & \rightarrow \text { Xev }) \simeq \frac{G_{F}^{2} m_{b}^{5}\left|V_{c b}\right|^{2}}{192 \pi^{3}} g\left(m_{c} / m_{b}\right) \\
& \times\left[1-(2 / 3 \pi) \alpha_{s}\left(m_{b}\right) f\left(m_{c} / m_{b}\right)\right],
\end{aligned}
$$

where:

$$
\begin{aligned}
& g(x)=1-8 x^{2}+8 x^{6}-x^{8}-24 x^{4} \ln x, \\
& f(x)=\left(\pi^{2}-31 / 4\right)(1-x)^{2}+3 / 2 .
\end{aligned}
$$

The decay width in the model can be evaluated as follows:

$$
\Gamma(b \rightarrow s \gamma) \simeq \frac{\alpha m_{b}^{5}}{(12 \pi)^{4}} \frac{\left|h_{2}^{\prime d^{*}} h_{3}^{\prime d}\right|^{2}}{M^{4}}
$$

Hence Equation (15) becomes:

$$
\begin{aligned}
& \operatorname{Br}(b \rightarrow s \gamma) \simeq \\
& \frac{\alpha G_{F}^{-2}\left|V_{c b}\right|^{-2} B r(b \rightarrow X e v)}{108 \pi g\left(m_{c} / m_{b}\right)\left[1-(2 / 3 \pi) \alpha_{s}\left(m_{b}\right) f\left(m_{c} / m_{b}\right)\right]} \frac{\left|h_{2}^{\prime d d^{*}} h_{3}^{\prime d}\right|^{2}}{M^{4}}
\end{aligned}
$$

Now taking $g\left(m_{c} / m_{b}\right) \approx 0.5, \quad f\left(m_{c} / m_{b}\right) \approx 2.5, \quad \alpha_{s}\left(m_{b}\right)$ $\approx 0.19, \alpha \approx 1 / 135, G_{F} \approx 10^{-5} \mathrm{GeV}^{-2}, V_{c b} \approx 0.04$ we have:

$B r(b \rightarrow s \gamma) \simeq 0.3\left(\frac{100 G e V}{M}\right)^{4}\left|h_{2}^{\prime d d^{*}} h_{3}^{\prime d}\right|^{2}$

The calculation in the SM for this branching is in good agreement with the experiments $B r(b \rightarrow s \gamma) \simeq 3.55 \times 10^{-4}$ (Nakamura et al., 2010). In our case, the new physics does not give contribution (i.e. does not contradict the SM result) if $M$ is of order 100 $\mathrm{GeV}$ and $h^{\prime d}<0.1$.

The flavor changing in the lepton sector is the same in the simplest seesaw extension of the SM. As such, it is to be noted that the charged lepton flavor violations such as $\mu \rightarrow e \gamma$ and $\mu \rightarrow 3 e$ are highly suppressed (Cheng and Li, 2003; He, 2003).

\section{Stable Anomalously-Charged Scalars}

As mentioned, the model contains two scalar doublets $\chi$ and $\phi$ with anomalous electric charges (besides heavier leptoquarks $j, k$ ). Therefore, one of the scalars may be very long-lived. Indeed, because of the electric charge conservation the lightest particle among them (assuming $\chi^{ \pm 1 / 6}$ ) never decays, even thought the quantum corrections up to any order of the perturbative theory are included. It is analogous to the case of a charged, lightest supersymmetric particle such as stau, stop, sbottom or lightest messenger in some gauge-mediated supersymmetry breaking scenario (Dimopoulos et al., 1996), where the stability is due to R-parity conservation. However, our proposal is different from the cases of the charged, next-to-lightest supersymmetric particles which can decay (Dimopoulos et al., 1997; Giudice and Rattazzi, 1999). 

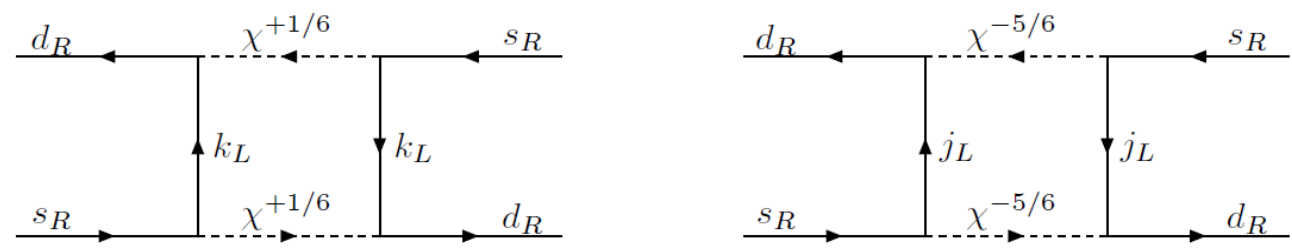

Fig. 1. Contributions to $K^{0}-\bar{K}^{0}$ mixing, where the vertices are proportional to $\mathrm{h}^{\text {'d }}$ and appropriate quark mixing matrix elements. Similarly, we have the box diagrams with the left-handed external quarks where $\phi$ gives also contributions
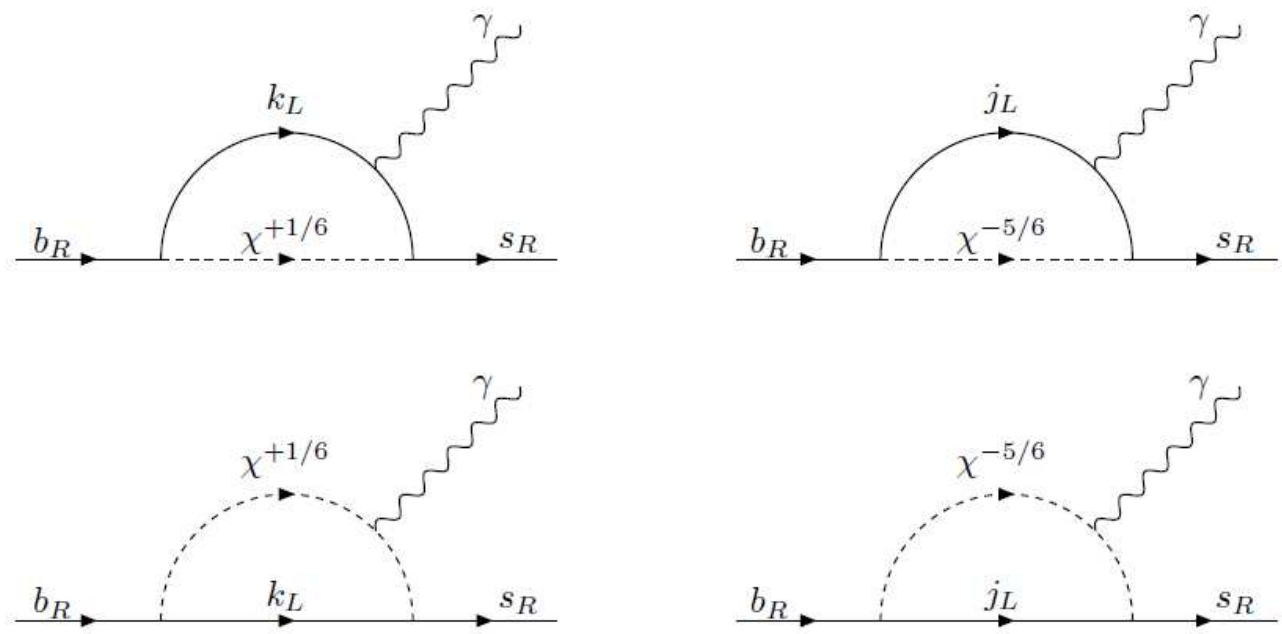

Fig. 2. Contributions to $\mathrm{b} \rightarrow \mathrm{s} \gamma$, where the vertices are proportional to $\mathrm{h}^{\text {'d }}$ and appropriate quark mixing matrix elements. Similarly, we have the diagrams with the left-handed external quarks where $\phi$ gives also contributions

If the stable scalar $\chi$ is produced at some collider or coming from cosmic rays, halo particles, or bulk matter (Perl, 2008; Perl et al., 2009; 2000; 2001; Ambrosio et al., 2000; Kim et al., 2007; Lee et al., 2002), it can easily escape from the detectors. Indeed, the general collider detectors cannot be used to find particles with an electric charge smaller than $1 / 3$ due to the uncertainty in trace reconstruction (Perl, 2008; Perl et al., 2009). The searches for its impinging on the earth due to the cosmic rays or halo particles have been sensitive only to the smallest charges of 1/5 (Perl et al., 2001; Ambrosio et al., 2000). The bulk matter and sea water searches (only with integer charges) (Smith and Bennett, 1979; and so forth, up to Chuzhoy and Kolb, 2009) have a similar sensitivity (Kim et al., 2007; Lee et al., 2002; Perl et al., 2000; 2001). There are also searches for millicharge particles with charges smaller than 0.1 , which are irrelevant (see, for a review, Perl, 2008). Note that the searches for the charged, next-to-lightest supersymmetric particles take the same status (Asaka et al., 2000; Heister et al., 2002; Aaltonen et al., 2009; Chen and Adams, 2010; LEP SUSY Working Group). Under this view, we conclude that there is currently no available experimental constraint applying to our case.

Assuming that $\chi$ is produced from the thermal bath of radiations in the early universe, it may lose energy due to the scattering with proton or electron when the universe cools down. If its velocity is smaller (larger) than the thermal average velocity of particles in the bath the scattering with proton (electron) dominates. For our galaxy with the average velocity $v_{\text {ave }} \sim 10^{-3} \mathrm{c}$, its relaxation timescale can be evaluated as (Rujula et al., 1990; Chuzhoy and Kolb, 2009):

$$
\tau_{\chi} \simeq\left\{\begin{array}{l}
8.5 \times 10^{15}\left(\frac{1}{q_{\chi}}\right)^{2}\left(\frac{M_{\chi}}{100 \mathrm{GeV}}\right)\left(\frac{v_{\chi}}{c}\right)^{3} \\
\times\left(\frac{n_{p}}{10^{-3} \mathrm{~cm}^{-3}}\right)^{-1} y r, \text { if } v_{\chi} \preceq v_{\text {ave }}, \\
5.5 \times 10^{12}\left(\frac{1}{q_{\chi}}\right)^{2}\left(\frac{M_{\chi}}{100 \mathrm{GeV}}\right)\left(\frac{v_{\chi}}{c}\right)^{3} \\
\times\left(\frac{n_{p}}{10^{-3} \mathrm{~cm}^{-3}}\right)^{-1} y r, \text { if } v_{\chi} \succeq v_{\text {ave }},
\end{array}\right.
$$

where, $M_{\chi}, v_{\chi}$ are the mass and velocity of $\chi$ respectively and $n_{p} \sim 10^{-3} \mathrm{~cm}^{-3}$ is the proton number density. For the scalar $\chi$ with a velocity in scale of the average one $v_{\chi} / c \sim 10^{-3}$, we find $\tau_{\chi} \sim 3 \times 10^{8}\left(M_{\chi} / 100\right.$ 
$\mathrm{GeV}) \mathrm{yr}$. Since the age of our galaxy is around $10^{9} \mathrm{yr}$, the scalar $\chi$ is in the galactic halo for $M_{\chi}>330 \mathrm{GeV}$. Otherwise, for $M_{\chi}<330 \mathrm{GeV}$, it is in the galactic disk. If the scalar $\chi$ is relativistic, for instance $v_{\chi} / c>0.1$, the relaxation timescale is $\tau_{\chi}>2 \times 10^{11}$ $\left(M_{\chi} / 100 \mathrm{GeV}\right)$ yr. Comparing with the galactic age leads to the fact that a relativistic $\chi$ with the mass $M_{\chi} \geq 1 \mathrm{GeV}$ always remains in the halo of galaxy.

Let us compute how many the scalars $\chi$ we can expect passing through the future designed detectors. Since the detectors are more sensitive with the more speed particles, we consider only the $\chi$ events coming from the halo. The flux of $\chi$ is (see Kudo and Yamaguchi, 2001):

$\phi_{\chi}=\frac{\Delta N_{\chi}}{\Delta A \Delta t}=\frac{\Delta N_{\chi}}{\Delta V} v_{\chi}=\frac{\rho_{\text {halo }} v_{\chi}}{M_{\chi}} f_{\chi}, \quad f_{\chi} \equiv \frac{\rho_{\chi}}{\rho_{\text {halo }}}$

where, $A, V, N_{\chi}, \rho_{\chi}$ and $\rho_{\text {halo }} \simeq 0.3 \mathrm{GeV} / \mathrm{cm}^{3}$ are area, volume, number of $\chi$ 's, mass densities of $\chi$ and galactic halo, respectively. Assuming that the local fraction $f_{\chi}$ nearby the earth traces its global fraction in the whole universe, it follows:

$f_{\chi} \simeq \frac{1}{2} \frac{\Omega_{\chi} h^{2}}{2 \Omega_{D M} h^{2}}$

where, the mass density of the dark matter is $\Omega_{D M} \simeq 0.35$ and $h \simeq 0.7$. Combining all, we have:

$\phi_{\chi} \simeq 2.6 \times 10^{8}\left(\frac{v_{\chi}}{c}\right)\left(\frac{100 G e V}{M_{\chi}}\right) \Omega_{\chi} h^{2} c m^{-2} s^{-1}$

The experiments will give $\phi_{\chi}$, which constrains the $\chi$ density $\Omega_{\chi} h^{2}$.

The ratio $\Omega_{\chi}$ of the $\chi$ mass density relative to the critical density of the universe can be obtained by solving the Boltzmann and Friedmann equations:

$$
\begin{aligned}
& \frac{d \rho_{I}}{d t}=-3 H \rho_{I}-\Gamma_{I} \rho_{I} \\
& \frac{d \rho_{R}}{d t}=-4 H \rho_{R}+\Gamma_{I} \rho_{I}+2\left\langle\sigma v_{\chi}\right\rangle\left\langle E_{\chi}\right\rangle\left[n_{\chi}^{2}-\left(n_{\chi}^{e q}\right)^{2}\right] \\
& \frac{d n_{\chi}}{d t}=-3 H n_{\chi}-\left\langle\sigma v_{\chi}\right\rangle\left[n_{\chi}^{2}-\left(n_{\chi}^{e q}\right)^{2}\right] \\
& H^{2} \equiv\left(\frac{\dot{a}}{a}\right)^{2}=\frac{8 \pi}{3 M_{P l}^{2}}\left(\rho_{I}+\rho_{R}+\rho_{\chi}\right)
\end{aligned}
$$

where, $I$ is an inflaton field (assume that the inflaton does not decay into $\chi), \rho_{I}$ and $\Gamma_{I}$ are the mass density and width respectively, $n_{\chi}\left(n_{\chi}^{e q}\right)$ is the number density (that of equilibrium), $\left\langle E_{\chi}\right\rangle$ is the thermal average of the $\chi$ energy, $\rho_{R}$ is the mass density of radiation, $H$ is the Hubble parameter and $\left\langle\sigma v_{\chi}\right\rangle$ is the thermalaveraged annihilation cross section times relative velocity. The dominant contributions to the annihilation cross section are $\chi \chi \rightarrow \gamma \gamma, Z \gamma$, which lead to:

$$
\left\langle\sigma v_{\chi}\right\rangle=\left(q_{\chi}\right)^{4} \frac{4 \pi \alpha^{2}}{M_{\chi}^{2}}+O\left(T / M_{\chi}\right)
$$

Due to the very small electric charge $q_{\chi}=1 / 6$, the annihilation cross section is always small even if the mass of $\chi$ as low as the electroweak scale. Therefore, the scalar never reaches a chemical equilibrium at the early stages. The analytic solution for the above equations has been known (see, for example, Giudice et al., 2001). It is noted that the $\Omega_{\chi} h^{2}$ is directly proportional to $\left\langle\sigma v_{\chi}\right\rangle$ since the scalar $\chi$ does not reach the equilibrium. The numerical interpretation was given by (Kudo and Yamaguchi, 2001), Fig. 1, which can be applied for our case with the constant contour lines now replaced by $\Omega_{\chi} h^{2} / q_{\chi}^{4} \simeq 1.3 \times 10^{3} \Omega_{\chi} h^{2}$. Taking $M_{\chi}=100-500 \mathrm{GeV}$, we have $1.3 \times 10^{3} \Omega_{\chi} h^{2} \leq 10^{-18}-2 \times 10^{-17}$. Using the figure, we naively obtain Table 1 for the upper limit of the reheat temperature:

$T_{R} \equiv\left(\frac{45}{4 \pi^{3}} \frac{1}{g_{*}\left(T_{R}\right)}\right)^{1 / 4} \sqrt{M_{P l}} \Gamma_{I}$

where, $g_{*}$ is the effectively relativistic degree of freedom.

To summarize, we remark that the bound on the $\chi$ mass in our model may be much lower than the other cases with the large charges (Rujula et al., 1990; Smith and Bennett, 1979). The typical lower bound on the mass of the long-lived massive particles in the various models other than may be as low as $1 \mathrm{TeV}$. However, in our case the $\mathrm{X}$ scalar can lie in the electroweak scale. This is in agreement with a search (Byrne et al., 2002) for the lower mass bound of the squarks and gluinos about $230 \mathrm{GeV}$. It is also in agreement with searches (Smith et al., 1982; Yamagata et al., 1993). The reheat temperature can be very low. This is in agreement with the other searches, for example, Kudo and Yamaguchi (2001) or the dark matter searches (Nakamura et al., 2010). 
Table 1. Naive upper limit for the reheat temperature

\begin{tabular}{llllll}
\hline $\mathrm{T}_{\mathrm{R}}(\mathrm{MeV})$ & 0.2 & 0.3 & 0.4 & 0.5 & 0.6 \\
\hline $\mathrm{M}_{\mathrm{x}}(\mathrm{GeV})$ & 100 & 200 & 300 & 400 & 500 \\
\hline
\end{tabular}

Finally, let us remind that the dark matter and stable charged particle searches can take a new life as recently shown in (Sanchez-Salcedo, 2010). There are stringent limits on the presence of massive charged particles because they might form heavy Hidrogen-like nuclei. However, it is noted that the sea water searches only constrain stable massive particles with integer charge (see also Sanchez-Salcedo, 2010) which is needed to recombine with electron or proton to make superheavy hydrogens or neutral CHAMPs. The stable scalars with anomalous charges as in our model are hard to have such recombinations. Of course, if had such combinations of $\chi$ scalars in nuclei to sum up an integer charge (however, it is impossible since these scalars are colorless, while the Coulomb forces always decouple them), the constraints should apply for the bound states of $\chi$ 's. To close this section, let us stress again that concerning the collider bounds indeed those can be evaded.

\section{Baryogenesis}

In this model the proton decay is discarded because the effective operator QQQL could not be generated with the provided model particle content. Also, the neutrinoless double beta decay with the effective operator $Q Q \bar{Q} \bar{Q} L L$ is explicitly suppressed. All these are the natural consequences due to the local lepton-number and baryon-number conservations. In the literature, the above processes are generally known to be prevented up to the very high scale as of the grand unification theories, where the lepton and baryon numbers may be violated. In our model the status is different. Although these processes cannot happen due to the gauge symmetries, there are still spontaneous breaking of baryon-number and lepton-number due to the VEVs of baryon-charged $\mathrm{S}_{\mathrm{B}}$ and lepton-charged $\mathrm{S}_{\mathrm{L}}$, respectively. And, the scales for these breakings in principle may be arbitrary but should be greater than the electroweak scale. These VEVs are just the sources for associated phenomena to be happened, independent of the explicit gauge symmetries.

As an example, the conservation of the lepton number and baryon number is the reason why we cannot generate the baryon number asymmetry as in the standard technics through explicit lepton/baryon violation interactions as such baryogenesis via the grand unification or baryogenesis via leptogenesis (Fukugita and Yanagida, 1986). The baryogenesis in this model may appear either one of the following cases:

We may realize a baryon asymmetry via the spontaneous symmetry breaking of the baryon number at the $\mathrm{TeV}$ scale due to $\mathrm{S}_{\mathrm{B}}$ as well as the ordinary quark products resulting from the decay of the unstable leptoquarks. The procedure for achieving the excess of baryon number can closely follow Harvey and Turner in (Harvey and Turner, 1990). The calculations in (Dulaney et al., 2011) in the models like ours have shown that this is possible.

Baryogenesis via leptogenesis: Let us recall that the neutrinos in our model gain the masses via the type I seesaw mechanism. The difference here is that the Majorana masses for the right-handed neutrinos are generated as a result from the spontaneous breaking of the lepton number due to $\mathrm{S}_{\mathrm{L}}$. The leptogenesis can be obtained via this source due to a nontrivial vacuum of the lepton number. The VEV for $S_{L}$ must be very high and the procedure for deriving an excess of lepton number, thus the baryon number, is similar to (Fukugita and Yanagida, 1986).

A detailed calculation for these cases to be included in the current work is out of the scope of this letter and we will devote it to a further publication.

\section{Other Aspects}

Our model has two new gauge bosons as mentioned $Z_{L}^{\prime}$ and $Z_{B}^{\prime}$, respectively gaining the masses via the lepton- and baryon-number breaking VEVs. If they are much heavier than the electroweak scale (particularly, this may exist in the second case of the baryogenesis as mentioned) their contributions to the collider phenomenology and affectations to the Higgs potential could be negligible. However, as shown above, in the first case the breaking of the baryon number at the $\mathrm{TeV}$ scale may be responsible for the baryogenesis. Then the $Z_{B}^{\prime}$ gets a mass in this scale. We can also have a $Z_{L}^{\prime}$ light as of $Z_{B}^{\prime}$ (this may only happen in the first case since the second case needs a very high scale of lepton number breaking). The $Z_{L}^{\prime}$ or $Z_{B}^{\prime}$ can then contribute to the known processes, e.g., $\quad e^{+} e^{-} \rightarrow Z_{L}^{\prime} \rightarrow \tau^{+} \tau^{-} \quad$ and $p p \rightarrow Z_{B}^{\prime} \rightarrow t \bar{t}$, or new processes to observe the lepton number violation $\mathrm{pp} \rightarrow \nu v$ provided that $Z_{L}^{\prime}$ and $Z_{B}^{\prime}$ mixing, which can be used to search for (for a detailed evaluation, see (Perez and Wise, 2010) and references therein).

At the LHC and ILC, we may have interesting processes due to the decays of the leptoquarks. Here we focus on the case where the leptoquarks decay into a stable scalar and a top quark. The channels are (i) LHC: $p p \rightarrow \overline{j j} \rightarrow \chi^{-1 / 6} \chi^{+1 / 6} \overline{t t}$, where the first process is possibly mediated by $\gamma, Z$, gluon, $Z_{B}^{\prime}$ (if $Z_{B}^{\prime}$ lies in TeV scale with its large enough gauge coupling, this contribution is also important) and (ii) ILC: $e^{+} e^{-} \rightarrow \overline{j j} \rightarrow \chi^{-1 / 6} \chi^{+1 / 6} \overline{t t}$, where the mediations of the first process may be $\gamma, Z, Z_{L}^{\prime}$ (if $Z_{L}^{\prime}$ lies in $\mathrm{TeV}$ scale and its gauge coupling is large enough). Finally, we can have typical processes with the 
stable scalar $\mathrm{x}^{ \pm 1 / 6}$ such as direct channel $\mathrm{e}^{+} \mathrm{e}^{-} \rightarrow \chi^{-1 / 6} \chi^{+1 / 6}$ at the ILC and $\gamma \gamma \rightarrow \chi^{-1 / 6} \chi^{+1 / 6}$ at the photon-photon collider. All the above processes are devoted to the forthcoming experimental considerations.

Let us note that the similar processes can happen in Tevatron and LEP, if the new particles are assumed to be light. The Tevatron and LEP then provide constraints on the model. Otherwise, those processes would been evaded if the new particles are much heavier than the electroweak scale. In this case the model is explicitly consistent with the effective theory and we have a natural seesaw mechanism for the neutrinos as well as the baryogenesis appearing in the mentioned second case, that all are quite similar to the standard context. Anyway, a constraint on the new physics at the TeV scale using the existing colliders are worth and a more detailed analysis on the model's consequences as briefly mentioned are needed. All these are large subjects out of scope of this letter. We will study of these issues to be published elsewhere in a near future.

In our model, with such a heavy leptoquark generation it is well-established that the gluon gluon fusion cross section for the SM Higgs is larger by a factor 9 (Djouadi, 2008). However, the new results from CDF and D0 do not rule out our model when the Higgs mass is $114 \mathrm{GeV}<\mathrm{M}_{\mathrm{H}}<120 \mathrm{GeV}$, or when $\mathrm{M}_{\mathrm{H}}>200 \mathrm{GeV}$ (Aaltonen et al., 2010). Notice that for a large mixing between $\mathrm{H}$ and the singlets $S_{L}$ and $S_{B}$ (if these scalars are assumed to be light enough) one can relax those constraints. These are similarities to the former proposal (Perez and Wise, 2010).

The scalar and vector leptoquarks as implied by the grand unified theories have been promising search candidates at the hadron colliders. Recently, there have been hints of scalar leptoquarks in the CMS data (see (CMS Collaboration; Queiroz et al., 2015; Bai and Berger, 2013) for the CMS result as well as for possible explanations). However, the leptoquarks in our model are fermions and obviously do not have any coupling to a quark and a lepton due to the Lorentz invariance (this also applies for other new particles). Therefore, our model predicts those channels (lepton-lepton-jetjet) with null results.

\section{Conclusion}

We have proposed a simple, predictive model of the gauged lepton and baryon charges. All the anomalies were removed by the presence of only the leptoquarks $\mathrm{j}^{+1 / 2}$ and $\mathrm{k}^{-1 / 2}$. The numbers of the observed lepton and quark families have been proved to be equal to the number of the fundamental colors, which is just three. Let us note that in the standard model the number of fermion families is left arbitrary and thus fail to answer this question. The right-handed neutrinos have been naturally existed as a requirement of the gravity-anomaly cancelation. This is an interesting feature in comparison with such particles as required for the $\mathrm{SO}(10)$ grand unification.

In contradiction to the previous proposals (Perez and Wise, 2010), the lepton sector in our model is minimal since it only assumes the mentioned right-handed neutrinos. The conservation of the lepton and baryon numbers will explicitly prevent the proton decay and neutrinoless double beta decay, but the spontaneous breaking of these charges will explain for associated low-energy phenomena such as neutrino mass and baryogenesis. Indeed, the small masses of the neutrinos have been explained by the type I seesaw mechanism in similarity to the simplest seesaw extension of the standard model. But, in our case the Majorana masses for the right-handed neutrinos get naturally generated as a result of spontaneous lepton-number breaking due to the VEV of lepton-charged $\mathrm{S}_{\mathrm{L}}$ scalar, although this symmetry is exact. A standard technic for the baryogenesis via leptogenesis is therefore followed. In other case, the spontaneous breaking of baryon number at $\mathrm{TeV}$ scale as well as the decay of the leptoquarks may also generate the baryogenesis. We particularly stress that the neutrino masses and leptogenesis as obtained in our model provide an insight into the standard contexts.

The flavor changing neutral current processes such as $K^{0}-\bar{K}^{0}$ mixing and $\mathrm{b} \rightarrow \mathrm{s} \gamma$ have been considered and evaluated. Here the new physics constrained is in natural consistency with the standard model. The model contains an interesting stable scalar as associated with an anomalous electric charge that can be very long-lived. This is similar to the supersymmetric models where the LSP is electrically charged such as stau, stop or sbottom. However, our scalar is different from the ones mentioned because it has very weak electromagnetic coupling. A naive evaluation has shown that the low bound on its mass may be in the electroweak scale and the reheat temperature can be in $\mathrm{MeV}$ order.

If the scales of lepton- and baryon-number breaking are much larger than the electroweak scale, the $\mathrm{S}_{\mathrm{L}}$ and $\mathrm{S}_{\mathrm{B}}$ would not affect the Higgs potential and the gauge bosons associated with these charges $Z_{L}^{\prime}$ and $Z_{B}^{\prime}$ do not contribute to the collider phenomenology. However, if these scales are as low as required in a case for the baryogenesis, they will take place. Also, existing as an answer of consistency of the model the new leptoquarks $\mathrm{j}, \mathrm{k}$ and the new scalars $\chi$ and $\phi$ with the anomalous electric charges (where almost the new particles fast decay, only one is the stable scalar as mentioned) have the interesting phenomenologies in the colliders such as the LHC, ILC and photon-photon collisions, which can be worth to search for. In addition, such similar processes can also happen in the existing accelerators such as the Tevatron and LEP, if the new particles are light enough, which provide constraints on the model. A detailed study on these issues are necessary to be published elsewhere. 


\section{Acknowledgment}

PVD would like to thank Dr. Do Thi Huong at Institute of Physics, Vietnam Academy of Science and Technology for discussions. This research is funded by Vietnam National Foundation for Science and Technology Development (NAFOSTED) under grant number 103.01-2013.43.

\section{Author's Contributions}

The issue has been proposed, computed and written by PVD, while essential physical background has been provided by HNL.

\section{Ethics}

This is an original work, containing unpublished material and supplied as arXiv:1010.3818 [hep-ph]. The corresponding author confirms that all the other authors have read and approved its publication with no ethical issues involved.

\section{Reference}

Nakamura, K., K. Hagiwara, K. Hikasa, H. Murayama and M. Tanabashi et al., 2010. Review of particle physics. J. Phys. G, 37: 075021-075021. DOI: $10.1088 / 0954-3899 / 37 / 7 \mathrm{~A} / 075021$

Foot, R., G.C. Joshi and H. Lew, 1989. Gauged baryon and lepton numbers. Phys. Rev. D, 40: 2487-2487. DOI: 10.1103/PhysRevD.40.2487

Carone, C.D. and H. Murayama, 1995. Third-family flavor physics in an $\mathrm{SU}(3) 3 \times \mathrm{SU}(2) \mathrm{L} \times \mathrm{U}(1) \mathrm{Y}$ model. Phys. Rev. D, 52: 4159-4159. DOI: 10.1103/PhysRevD.52.4159

Perez, P.F. and M.B. Wise, 2010. Baryon and lepton number as local gauge symmetries. Phys. Rev. D, 82: 079901-079901. DOI: 10.1103/PhysRevD.82.011901

Dulaney, T.R., P.F. Perez and M.B. Wise, 2011. Dark matter, baryon asymmetry and spontaneous B and L breaking. Phys. Rev. D, 83: 023520-023520. DOI: 10.1103/PhysRevD.83.023520

Chao, W., 2010. Neutrino masses and a TeV scale seesaw mechanism. Phys. Rev. D, 82: 016008016008. DOI: 10.1103/PhysRevD.82.016008

Ko, P. and Y. Omura, 2011. Supersymmetric U(1)B x $\mathrm{U}(1) \mathrm{L}$ model with leptophilic and leptophobic cold dark matters. Phys. Lett. B, 701: 363-366. DOI: $10.1016 /$ j.physletb.2011.06.009

Kelso, C., H.N. Long, R. Martinez and F.S. Queiroz, 2014. Connection of $\mathrm{g}-2 \mu$, electroweak, dark matter and collider constraints on 331 models. Phys. Rev. D, 90: 113011-113011. DOI: 10.1103/PhysRevD.90.113011

Cogollo, D., A.X. Gonzalez-Morales, F.S. Queiroz and P.R. Teles, 2014. Excluding the light dark matter window of a 331 model using LHC and direct dark matter detection data. JCAP, 11: 002. DOI: $10.1088 / 1475-7516 / 2014 / 11 / 002$
Dong, P.V., N.T.K. Ngan and D.V. Soa, 2014. Simple 33-1 model and implication for dark matter. Phys. Rev. D, 90: 075019-075019. DOI: 10.1103/PhysRevD.90.075019

Dong, P.V., T.P. Nguyen and D.V. Soa, 2013. 3-3-1 model with inert scalar triplet. Phys. Rev. D, 88: 095014095014. DOI: 10.1103/PhysRevD.88.095014

Dong, P.V., H.N. Long and H.T. Hung, 2012. Question of Peccei-Quinn symmetry and quark masses in the economical 3-3-1 model. Phys. Rev. D, 86: 033002-033002. DOI: $10.1103 /$ PhysRevD.86.033002

Rujula, D.A., S.L. Glashow and U. Sarid, 1990. Charged dark matter. Nucl. Phys. B, 333: 173-194. DOI: 10.1016/0550-3213(90)90227-5

Dimopoulos, S., D. Eichler, R. Esmailzadeh and G.D. Starkman, 1990. Getting a charge out of dark matter. Phys. Rev. D, 41: 2388-2388. DOI: 10.1103/PhysRevD.41.2388

Smith, P.F. and J.R. Bennett, 1979. A search for heavy stable particles. Nucl. Phys. B, 149: 525-533. DOI: 10.1016/0550-3213(79)90006-3

Smith, P.F., J.R.J. Bennett, G.J. Homer, J.D. Lewin and H.E. Walford et al., 1982. A Search For Anomalous Hydrogen In Enriched D-2 O, Using A Timeofflight Spectrometer. Nucl. Phys. B, 206: 333-333. DOI: 10.1016/0550-3213(82)90271-1

Yamagata, T., Y. Takamori and H. Utsunomiya, 1993. Search for anomalously heavy hydrogen in deep sea water at 4000-m. Phys. Rev. D, 47: 1231-1234. DOI: $10.1103 /$ PhysRevD.47.1231

Giudice, G.F., E.W. Kolb and A. Riotto, 2001. Largest temperature of the radiation era and its cosmological implications. Phys. Rev. D, 64: 023508-023508. DOI: 10.1103/PhysRevD.64.023508

Kudo, A. and M. Yamaguchi, 2001. Inflation with low reheat temperature and cosmological constraint on stable charged massive particles. Phys. Lett. B, 516: 151-155. DOI: 10.1016/S0370-2693(01)00938-8

Byrne, M., C. Kolda and P. Regan, 2002. Bounds on charged, stable superpartners from cosmic ray production. Phys. Rev. D, 66: 075007-075007. DOI: 10.1103/PhysRevD.66.075007

Chuzhoy, L. and E.W. Kolb, 2009. Reopening the window on charged dark matter. JCAP 0907, 014. DOI: 10.1088/1475-7516/2009/07/014

Asaka, T., K. Hamaguchi and K. Suzuki, 2000. Cosmological gravitino problem in gauge-mediated supersymmetry breaking models. Phys. Lett. B, 490: 136-146. DOI: 10.1016/S0370-2693(00)00959-X

Heister, A., 2002. Search for scalar quarks in $\mathrm{e}+\mathrm{e}-$ collisions at $\mathrm{s}$ up to $209-\mathrm{GeV}$. Phys. Lett. B, 537: 520. DOI: $10.1016 / \mathrm{S} 0370-2693(02) 01827-0$

Aaltonen, T., J.A. Adelman, T. Akimoto, B.A. Gonzalez and S. Amerio et al., 2009. Search for long-lived massive charged particles in $1.96 \mathrm{TeV}$ pp collisions. Phys. Rev. Lett., 103: 021802-021802.

DOI: 10.1103/PhysRevLett.103.021802 
Chen, J. and T. Adams, 2010. Searching for high speed long-lived charged massive particles at the LHC. Eur. Phys. J. C, 67: 335-342. DOI: 10.1140/epjc/s10052-010-1283-9

Greub, C., T. Hurth and D. Wyler, 1996. Virtual O(Ts) corrections to the inclusive decay b s. Phys. Rev. D, 54: 3350-3350. DOI: 10.1103/PhysRevD.54.3350

Cheng, T.P. and L.F. Li, 2003. M e in Theories with dirac and majorana neutrino-mass terms. Phys. Rev. Lett., 45: 1908-1908. DOI: 10.1103/PhysRevLett.45.1908

He, B., T.P. Cheng and L. Ling-Fong, 2003. Less suppressed $\mu \mathrm{eU}$ and $\mathrm{VW} \mu$ loop amplitudes and extra dimension theories. Phys. Lett. B, 553: $277-$ 283. DOI: 10.1016/S0370-2693(02)03258-6

Dimopoulos, S., G.F. Giudice and A. Pomarol, 1996. Dark matter in theories of gauge-mediated supersymmetry breaking. Phys. Lett. B, 389: 37-42. DOI: 10.1016/S0370-2693(96)01241-5

Dimopoulos, S., S. Thomass and J.D. Wells, 1997. Sparticle spectroscopy and electroweak symmetry breaking with gauge-mediated supersymmetry breaking. Nucl. Phys. B, 488: 39-91. DOI: 10.1016/S0550-3213(97)00030-8

Giudice, G.F. and R. Rattazzi, 1999. Theories with gauge mediated supersymmetry breaking. Phys. Rept., 322: 419-499. DOI: $10.1016 / \mathrm{S} 0370-1573(99) 00042-3$

Perl, M.L., 2008. Searches for Fractionally charged particles: What should be done next?. Nuclear Physics B Proc. Supplements, 189: 5-8. DOI: 10.1016/j.nuclphysbps.2009.03.002

Perl, M.L., E.R. Lee and D. Loomba, 2009. Searches for fractionally charged particles. Ann. Rev. Nucl. Part. Sci., 59: 47-65. DOI: 10.1146/annurev-nucl-121908-122035

Perl, M.L., P.C. Kim, V. Halyo, E.R. Lee and I.T. Lee et al., 2001. The search for stable, massive, elementary particles. Int. J. Mod. Phys. A, 16: 2137-2164. DOI: $10.1142 / \mathrm{S} 0217751 \mathrm{X} 01003548$

Ambrosio, M., R. Antolini, G. Auriemma, D. Bakari and A. Baldini et al., 2000. A Search for lightly ionizing particles with the MACRO detector. Phys. Rev. D, 62: 052003-052003. DOI: 10.1103/PhysRevD.62.052003

Kim, C., Y. Liu, A. Kühnle, S. Hess and S. Viereck et al., 2007. Gravitational stability of suspensions of attractive colloidal particles. Phys. Rev. Lett., 99: 028303-028303.

DOI: 10.1103/PhysRevLett.99.028303

Lee, I.T., S. Fan, V. Halyo, E.R. Lee and P.C. Kim et al., 2002. Large bulk matter search for fractional charge particles. Phys. Rev. D, 66: 012002. DOI: 10.1103/PhysRevD.66.012002

Perl, M.L., V. Halyo, P.C. Kim, E.R. Lee and I.T. Lee et al., 2000. The search for fractional charge elementary particles and very massive particles in bulk matter.
Harvey, J.A. and M.S. Turner, 1990. Cosmological baryon and lepton number in the presence of electroweak fermion-number violation. Phys. Rev. D, 42: 33443344. DOI: 10.1103/PhysRevD.42.3344

Fukugita, M. and T. Yanagida, 1986. Barygenesis without grand unification. Phys. Lett. B, 174: 45-47. DOI: 10.1016/0370-2693(86)91126-3

Djouadi, A., 2008. The anatomy of electroweak symmetry breaking: Tome I: The higgs boson in the standard model. Phys. Rept., 457: 1-216. DOI: $10.1016 /$ j.physrep.2007.10.004

Aaltonen, T., V.M. Abazov, B. Abbott, M. Abolins and B.S. Acharya et al., 2010. Combination of tevatron searches for the standard model higgs boson in the $\mathrm{W}+\mathrm{W}$-decay mode. Phys. Rev. Lett., 104: 061802-061802.

DOI: 10.1103/PhysRevLett.104.061802

Queiroz, F.S., K. Sinha and A. Strumia, 2015. Leptoquarks, dark matter and anomalous LHC events. Phys. Rev. D, 91: 035006-035006. DOI: 10.1103/PhysRevD.91.035006

Bai, Y. and J. Berger, 2013. Fermion portal dark matter. Cornell University Library.

Sanchez-Salcedo, F.J., 2010. The survival of dynamical fossils in dwarf spheroidal galaxies in conventional and modified dynamics. Mon. Not. Roy. Astron. Soc., 407: 1135-1135.

DOI: $10.1111 /$ j.1365-2966.2010.16963.x 